Erratum: Pseudo-Hermiticity for a class of nondiagonalizable Hamiltonians [J. Math. Phys. 43, 6343 (2002)]

Ali Mostafazadeh

Citation: Journal of Mathematical Physics 44, 943 (2003); doi: 10.1063/1.1540714

View online: http://dx.doi.org/10.1063/1.1540714

View Table of Contents: http://aip.scitation.org/toc/jmp/44/2

Published by the American Institute of Physics

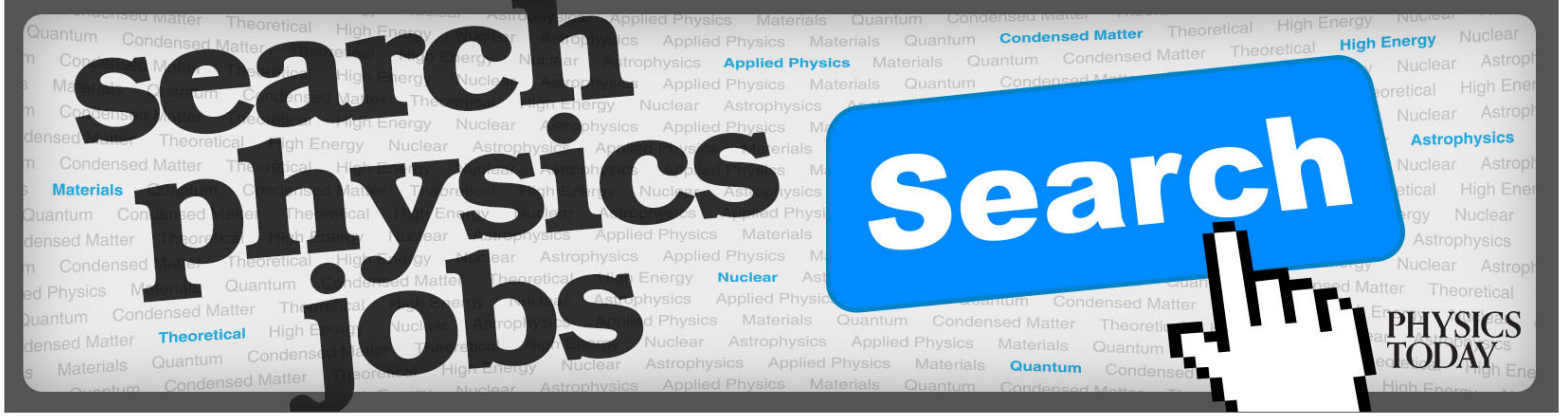




\title{
Erratum: Pseudo-Hermiticity for a class of nondiagonalizable Hamiltonians [J. Math. Phys. 43, 6343 (2002)]
}

\author{
Ali Mostafazadeh ${ }^{\mathrm{a}}$ \\ Department of Mathematics, Koç University, \\ Rumelifeneri Yolu, 34450 Sariyer, Istanbul, Turkey
}

[DOI: $10.1063 / 1.1540714$ ]

Recently, the authors of Ref. 1 used the framework provided in Ref. 2 to re-examine the consequences of pseudo-Hermiticity for the class of block-diagonalizable Hamiltonians introduced in Ref. 2. In doing so, they discovered that Theorem 2 of Ref. 2 did not hold, as they could find a counter-example. This theorem must be replaced with the following.

Theorem 2: Let $H$ be as in Theorem 1 of Ref. 2. Then $H$ is pseudo-Hermitian if and only if it is Hermitian with respect to an inner product $\langle\langle\rangle$,$\rangle that supports a positive-semidefinite basis { }^{3}$ including the eigenvectors of $H$. In particular, for every eigenvector $\psi$ of $H,\langle\langle\psi \mid \psi\rangle\rangle \geqslant 0$; if the corresponding eigenvalue is real and nondefective (algebraic and geometric multiplicities are equal), $\langle\langle\psi \mid \psi\rangle\rangle\rangle>0$; otherwise $\langle\langle\psi \mid \psi\rangle\rangle=0$.

Proof: As shown in Ref. 2, pseudo-Hermiticity of $H$ implies that $H$ is Hermitian with respect to the inner product $\langle\langle,\rangle\rangle_{\eta}$ with $\eta$ given by Eq. (15) of Ref. 2 and $\sigma_{\nu_{0}, a}=1$. It is not difficult to check that indeed the basis vectors $\left|\psi_{n}, a, i\right\rangle$, constructed in Ref. 2, have the property that $\left\langle\left\langle\psi_{n}, a, i \mid \psi_{n}, a, i\right\rangle\right\rangle \geqslant 0$, and that $\left\langle\left\langle\psi_{n}, a, i \mid \psi_{n}, a, i\right\rangle\right\rangle>0$ only for the cases that $p_{n, a}=1$ and $E_{n}$ $\in \mathbb{R}$, i.e., $\left|\psi_{n}, a, i=1\right\rangle$ is an eigenvector of $H$ with a real eigenvalue. Furthermore, by construction, this basis includes all the eigenvectors of $H$. The proof of the converse is the same as the one given in Ref. 2.

It is important to note that having a positive-semidefinite basis does not imply that the inner product $\langle\langle,\rangle\rangle_{\eta}$ is positive-semidefinite (unless the Hamiltonian is diagonalizable and has a real spectrum in which case both the basis and the inner product $\langle\langle,\rangle\rangle_{\eta}$ are positive definite. ${ }^{4}$ ) If the Hamiltonian has defective or complex(-conjugate pair(s) of) eigenvalues, there will always be at least two null vectors with negative ${ }^{3}$ linear combinations. Unlike positive vectors, linear combinations of nonnegative vectors need not be nonnegative.

${ }^{1}$ G. Scolarici and L. Solombrino, "On the pseudo-Hermitian nondiagonalizable Hamiltonians," arXiv:quant-ph/0211161. ${ }^{2}$ A. Mostafazadeh, J. Math. Phys. 43, 6343 (2002).

${ }^{3} \mathrm{~A}$ vector $\phi$ is respectively said to be positive, null (zero), negative, if $\langle\langle\phi \mid \phi\rangle\rangle>0,\langle\langle\phi \mid \phi\rangle\rangle=0,\langle\langle\phi \mid \phi\rangle\rangle\langle 0$. It is said to be nonnegative if $\langle\langle\phi \mid \phi\rangle\rangle \geqslant 0$. A basis is called positive-semidefinite if it consists of nonnegative vectors. See, for example, J. Bognar, Indefinite Inner Product Spaces (Springer, Berlin, 1974).

${ }^{4}$ A. Mostafazadeh, J. Math. Phys. 43, 2814 (2002).

${ }^{\text {a)} E l e c t r o n i c ~ m a i l: ~ a m o s t a f a z a d e h @ k u . e d u . t r ~}$ 